\title{
La formación del contrato electrónico en el marco de la Comunidad Andina*
}

\author{
The electronic contract formation in the framework of the \\ Andean Community \\ Recibido: 18 de mayo de 2012 - Revisado: 06 de junio de 2012 - Aceptado: 13 de julio de 2012
}

William David Hernández Martínez ${ }^{* *}$

\section{Resumen}

La influencia de las Tecnologías de la Información y las Comunicaciones (TIC's) en los todos los aspectos de la sociedad es un hecho incuestionable que implica, para el derecho, la responsabilidad ineludible de propiciar el cumplimiento de las declaraciones o finalidades de la Sociedad de la Información. El mundo actual, enmarcado en un proceso de globalización e integración regional, se encamina hacia a la armonización normativa. En consonancia con lo anterior, el presente documento estudia los elementos que fundamentan la unificación normativa formación del contrato por medios electrónicos en la Comunidad Andina.

\section{Palabras clave}

Armonización, Unificación, Comunidad Andina, Contratación Electrónica, formación del contrato.

\footnotetext{
Abstract

The influence of the Information and Communication Technology (ICT) in all the aspects of the society is an unquestionable fact that implies, for the Law, the inescapable responsibility of fostering the fulfillment of the declarations or objectives of the Society of Information. Today's world, framed in a process of globalization and regional integration, heads to the normative harmonization. In line with the above, the present document studies the elements supporting the normative unification concerning the formation of the contract by electronic means in the Andean Community.

Key words

Harmonization, Unification, Andean Community, Electronic Contracting, Contract formation.
}

\footnotetext{
${ }^{*}$ Resultado del proyecto de investigación titulado La contratación por medios electrónicos en el marco de la Comunidad Andina, financiado por el Grupo de Investigación en Derecho Privado-USA. Adscrito a la Escuela de Derecho de la Universidad Sergio Arboleda.

** Abogado y Magíster en Derecho de la Universidad Sergio Arboleda. Profesor e investigador asistente de la misma institución, adscrito al Departamento de Derecho Privado y al Grupo de Investigación en Derecho Privado-USA.

Correo electrónico:

william. hernandez@usa.edu.co
} 


\section{Introducción}

Los contextos sociales del mundo actual se encuentran sometidos a constantes cambios debido a los nuevos paradigmas formulados dentro del proceso de evolución de la humanidad. En este sentido, las modificaciones desde la ciencia, la economía, la política o el derecho influyen necesariamente en la interpretación de los preceptos preestablecidos y exigen de los actores de las diferentes ciencias un esfuerzo en la "relectura" de las instituciones y de los conceptos, así como la formulación de nuevas "teorías" que soporten y doten de seguridad y certeza los avances de la humanidad.

El marco del mundo actual nos muestra los efectos de la globalización y de las relaciones económicas heterogéneas con tendencia a la integración regional como elemento generador de ventajas competitivas y cooperativas (Zarur Miranda, 2008),en donde los procesos de armonización y unificación se erigen como elementos esenciales. A su turno, el acelerado e imparable avance de las Tecnologías de la Información y las Comunicaciones (TIC) y su, cada vez mayor, empleo en las relaciones comerciales (de diferentes índoles) demandan el análisis de los conceptos relativos a la contratación electrónica desde una perspectiva supranacional, consciente de que el futuro está en la integración regional al amparo de la actual sociedad global del conocimiento.

Así las cosas, el presente artículo busca analizar las corrientes teóricas pertinentes y necesarias a efectos de determinar los elementos normativos que posibiliten la armonización en relación con la formación del contrato mercantil electrónico a nivel de la Comunidad Andina de Naciones. Para ello se encuentra divido en cuatro acápites a saber: 1 . El planteamiento del problema de investigación. 2. Descripción de la metodología a emplear. 3. El estudio de la formación del contrato desde una visión de derecho comparado a partir de los aspectos que se consideran de mayor relevancia en torno a la oferta y a la aceptación. 4) Algunas conclusiones sobre el particular.

\section{Problema de investigación}

Sostener que las TIC han incidido en todos los aspectos de las sociedades (económicas, sociales, políticas, entre otros) no es una novedad. Sin embargo, la dinamicidad y especialidad de las TIC implican la responsabilidad ineludible del derecho, consistente en la búsqueda de los escenarios que doten de certeza las manifestaciones contractuales que se originan en la Sociedad de la Información (Cumbre Mundial sobre la Sociedad de la Información, 2004) o, en otras palabras, de contrarrestar las inquietudes y vicisitudes que minan la seguridad de los ordenamientos jurídicos (Reusser, 2003).

Los contratos electrónicos como elemento integrante del denominado comercio electrónico, es decir aquellos en los cuales se emplean sistemas electrónicos de procedimiento y transmisión de información para celebrar, e incluso ejecutar, el contrato sea cual sea el tipo de contrato y el objeto del mismo (Madrid Parra, 2010, p. 188; Remolina, 2006, p. 2; Rincón, 2004; 2006b, pp. 19-23), no son ajenos a esta realidad, esto ha suscitado al interior de los Estados la necesidad de establecer un marco normativo más o menos amplio (Olivera \& Proto, 2006).

La regulación sobre el comercio electrónico, en general, y la contratación electrónica, en especial, mantienen la tendencia de la lex mercatoria moderna, entendida como un orden jurídico que insta a la autorregulación, en otras palabras, a la posibilidad de los agentes del comercio internacional de reemplazar la ley por el contrato, el juez por el arbitraje (Galgano, 2005, p. 105$)^{1}$, y por tanto que se caracteriza por su anacionalidad (Fernández, 2007, pp. 57-58; 2004, pp. 95-96; 2000).

No obstante lo anterior, se ha de tener en cuenta que las reglas de derecho aplicable a las diferentes relaciones o vínculos jurídicos surgen 
de los mismos comerciantes, quienes a través de sus usos y prácticas ya han comenzado a crear costumbres. En otras palabras, las instituciones legales aplicables a los negocios y contratos mercantiles encuentran su origen en los usos y prácticas de la sociedad de los comerciantes, hoy considerados desde la perspectiva del concepto de empresa (Galgano, 2005, p. 35; García, 2006), siendo función del legislador, entonces, dotar de estabilidad, certeza y generalidad las bases dictadas por la praxis social (Corte Constitucional, 1993, Sentencia C-486, p. 8, consid. 5).

En desarrollo de lo anterior, se resalta el trabajo del grupo de trabajo de prácticas en el comercio electrónico de la Cámara de Comercio Internacional al presentar el borrador de las Reglas Uniformes y Guías para el Comercio y Pago Electrónico (Urgets), mediante las cuales se recogen las costumbres en aras de uniformizar los contratos electrónicos (Calderón, 2000; Cibertribunal Peruano, 2008), así como la labor de Uncitral, mediante la formulación de la Ley Modelo de Comercio Electrónico (LMCE), que emerge como fundamento de la normatividad sobre la materia expedida en los últimos años en los diferentes estados (Iriarte Ahon, 2005) ${ }^{2}$.

Ahora bien, la formulación de políticas y normas respecto del e-business acusa una gran variedad de problemas, entre los cuales se pueden señalar la indeterminación geográfica de las partes, la desconfianza del usuario en las transacciones, la falta de protección del consumidor, entre otros (Iriarte Ahon, 2006). En torno a estos interrogantes se destaca la necesidad de poseer estándares básicos que doten de plena validez a los actos, contratos y negocios que surgen en torno de las TIC a nivel mundial y, en consecuencia, a proyectar sobre los actores la seguridad jurídica ${ }^{3}$ necesaria en este tipo de transacciones, a veces denominada lex retialis (Olivera, 2008, p. 8; Grün, 1995).

A nivel andino, los países han declarado abiertamente su compromiso de constituir como objetivos comunes: i) el garantizar el acceso de la población a los servicios de telecomunicaciones, y ii) establecer normas que doten de seguridad las diferentes fases de las operaciones comerciales que utilizan recursos electrónicos. Sin embargo, la situación comunitaria no es alentadora, principalmente porque las tendencias, orientaciones y disposiciones son divergentes (Iriarte Ahon, 2005, p. 5), lo que propicia la inseguridad jurídica en la materia.

Por otra parte, tal y como se puede comprobar en los estudios nacionales, la regulación estatal acusa el problema de establecer un sistema de normas que atienda una necesidad particular e individual, una necesidad nacional, bajo un esquema de espacio-tiempo limitado, que deje de lado la internacionalidad como elemento sustancialmente y característica inherente al comercio electrónico. En oposición a la realidad del ambiente comunitario, el presente proyecto de investigación se centra en la necesidad de poseer normas con vocación internacional que eliminen la disparidad de los ordenamientos nacionales a través de la armonización o la unificación normativa y que reconozca lo cambiante de las situaciones particulares propias del comercio electrónico.

La búsqueda de una normatividad andina común, respecto a la contratación por medios electrónicos parece haberse quedado en pronunciamientos, políticas, objetivos o finalidades, lo cual obliga a las políticas estatales a decaer u ocuparse en los problemas básicos de infraestructura, en oposición al debate que, principalmente en Europa y Estados Unidos, se ha centrado en temas avanzados como la privacidad y confianza del consumidor y la legalidad de las actividades digitales. De acuerdo con las consideraciones anteriores se evidencia la necesidad de armonizar o unificar la normativa respecto al entorno digital, de establecer principios y medidas dentro de las organizaciones regionales existentes, en el caso latinoamericano la Comunidad Andina (CAN), que permitan dotar de seguridad jurídica a los actores de las relaciones electrónicas (Silveira, 2001). 
De esta necesidad de armonización y unificación surge el objeto propio de esta investigación, sintetizado metodológicamente en la siguiente pregunta jurídica: ¿Cuáles son los aspectos sustantivos a partir de los cuales es posible armonizar la regulación de la formación del contrato electrónico en la Comunidad Andina?

\section{Metodología}

El trabajo planteado es una investigación básica jurídica que tiene por objeto el estudio de las normas jurídicas, en sentido amplio, que sobre la formación del contrato por medios electrónicos se han promulgado en los países miembros de la CAN (Bolivia, Colombia, Ecuador y Perú), así como la jurisprudencia y doctrina que sobre el particular se profiera, en aras a contribuir en la formulación de principios y reglas de validez con aplicación general, inspirados en la experiencia de la Unión Europea, que se integren al Derecho Uniforme del Comercio Internacional (DUCI), de tal forma que los hallazgos sirvan como sustento de proyectos de unificación y armonización (Fernández Rozas, 2007, pp. 59-62; 2004, pp. 97-100; 2000).

La investigación propuesta se orienta en la búsqueda de aspectos comunes en la formación del contrato, respecto de un elemento específico que es el uso de las TIC, partiendo de la base del proceso de regionalización e integración llevado a cabo en la Comunidad Andina de Naciones, sin olvidar que en la actualidad se reconoce la existencia de un nuevo ciudadano, el ciudadano económico (Galgano, 2005, p. 35; Moncayo, 2004, p. 132), que se resiste a ser considerada como objeto jurídico y demanda un papel o rol en la economía global, fomentando la autorregulación.

\section{La formación del contrato}

De forma genérica, es posible definir el contrato como la institución social y jurídica (Mantilla Espinosa, 2007, p. 79) que surge del acuerdo de voluntades, entre dos o más sujetos, para crear, modificar o extinguir vínculos jurídicos (Código Civil del Estado Plurinacional de Bolivia (CCBol), artículo 450. Código Civil de la República de Colombia (CCC), artículo 1495. Código de Comercio de la República de Colombia (CCoC), artículo 864. Código Civil de la República del Ecuador (CCE), artículo 1454. Código civil de la República del Perú (CCP), artículo 1351).

Ahora bien, en el punto específico de la formación del contrato electrónico se hace referencia a las normas aplicables a la coincidencia de las voluntades negociables (tradicionalmente analizadas a partir de la oferta y la aceptación), dentro de las cuales se pueden entender incorporado el deber de información posterior a la celebración del contrato (Davara, 2005). En el presente acápite se analizan los aspectos que se consideran de mayor relevancia o incidencia en la formación del contrato electrónico desde una perspectiva de derecho comparado en el marco de la CAN, con referencia a algunos elementos de carácter internacional que, desde hace ya varios años, hacen parte de la lex mercatoria moderna ${ }^{4}$.

\section{Manifestación de la voluntad por un me- dio electrónico}

En el estudio de lo que considera como el iter contractual, los diferentes ordenamientos jurídicos nacionales (de las diferentes familias jurídicas), así como los elementos normativos de carácter internacional, reconocen la aplicación y eficacia de diferentes principios en los contratos, los cuales se consideran igualmente aplicables a la contratación electrónica, tales como la i) autonomía de la voluntad privada, ii) la buena fe, iii) la consensualidad y iv) la fuerza obligatoria de los negocios jurídicos.

Ahora bien, sin pretender negar la importancia de los demás principios, es válido afirmar que el principio de consensualidad se erige, sin lugar a dudas, como un presupuesto necesario para el reconocimiento de efectos jurídicos a la 
contratación por medios electrónicos. En este sentido, la regla general es el reconocimiento de la libertad de forma en la manifestación de la voluntad de contratar, manteniendo las solemnidades sustanciales para algunos contratos específicos, a título de excepción.

La consensualidad ha sido reconocida a nivel internacional por los Principios sobre los Contratos Comerciales Internacionales de Unidroit (en adelante los Principios) (López Rodríguez, 2006, p. 119-122; Unidroit, 2004)5, instrumento que reconoce en el artículo 1.2, que ninguna de las disposiciones del aludido cuerpo normativo requiere que la manifestación de voluntad sea expresada o probada en una forma en particular, esto es, que un contrato puede ser celebrado y probado en cualquier medio. $\mathrm{La}$ abierta prescripción del artículo 1.2 de los principios ha de entenderse armónico con el artículo 1.4 en el cual se expone que dicho instrumento no restringe la aplicación de las normas de carácter imperativo del orden nacional. Es así como, para el instrumento en estudio, la regla general es la consensualidad en la formación de los contratos, admitiendo la posibilidad de que, en determinados casos, el contrato internacional se someta a las formalidades que señalen las partes, el derecho nacional o los tratados internacionales (Oviedo et al., 1999; Alvarado Herrera et al., 1999, pp. 53-58).

La interpretación de la consensualidad acogida por los Principios se muestra plenamente coherente con lo dispuesto en la Convención de las Naciones Unidas sobre Compraventa Internacional de Mercaderías (en adelante Convención o CISG) (Corte Constitucional, 2000, C-529; Oviedo Albán, 2001; Perales Viscasillas, 2001) que, en su artículo 11, dispone que el contrato de compraventa no tendrá que celebrarse ni probarse por escrito ni estará sujeto a ningún otro requisito de forma ${ }^{6}$.

Para Crucilaegui (2005, p. 129) la previsión contenida en el artículo 11 de la CISG no es de carácter imperativo, razón por la cual "las partes pueden acordar otros requisitos en lo que se refiere a la forma del contrato de manera expresa", en aplicación de la amplia potestad otorgada por el artículo 6 del mismo instrumento.

Por otra parte, al revisar la normatividad comunitaria europea, y en especial lo dispuesto por la Directiva 2000/31/CE (en adelante DCE), se destaca la disposición que se orienta a imponer a los Estados miembros la obligación de velar porque su legislación permita la celebración de contratos por vía electrónica, para lo cual garantizarán, en particular, que el régimen jurídico aplicable al proceso contractual no entorpezca la utilización real de los contratos por vía electrónica, "ni conduzca a privar de efecto y validez jurídica a este tipo de contratos en razón de su celebración por vía electrónica" (DCE, artículo 9.1; Espinosa Quintero, 2008) ${ }^{7}$.

Desde la perspectiva de los estados integrantes de la CAN se destaca el reconocimiento unánime del principio de consensualidad en los diferentes ordenamientos, tal y como se observa en la innegable coherencia y semejanza de las disposiciones normativas ${ }^{8}$ que tratan el tema, tales como: el artículo 521 del Código Civil de Bolivia (CCBol), el artículo 787 del Código de Comercio de Bolivia (CCoBol), el artículo 824 del Código de Comercio de Colombia (CCoC) y el artículo 1352 del Código Civil de Perú $(\mathrm{CCP})^{9}$.

De lo anterior se concluye que, tanto para los instrumentos internacionales como para los ordenamientos jurídicos nacionales de los estados miembros de la CAN, la voluntad de contratar y de obligarse se puede expresar verbalmente o por cualquier otro medio inequívocamente dirigido a tal fin (lo cual incluye, per se, los medios electrónicos), salvo que una norma imperativa exija una solemnidad especial como requisito esencial para la validez del contrato, en cuyo caso este no se perfecciona sino hasta cuando la mencionada solemnidad sea satisfecha. 


\section{Oferta}

Es tradicional a los diferentes sistemas jurídicos que los contratos, en especial los mercantiles, se formen como resultado de un proceso de negociación, en el cual los futuros co-contratantes definen el contenido, alcance y efectos de su contrato, teniendo en cuenta, en todo caso, sus necesidades y posibilidades de ejecución (Alvarado Herrera et al., 1999, p. 97). Es así como resulta sencillo establecer o determinar el iter contractual a partir del cruce o intercambio de propuestas de negocio (ofertas y contraofertas) que culmina con una aceptación, con la cual se perfecciona, finalmente, el acuerdo de voluntades obligacionales de las partes y dan lugar a la existencia del contrato.

La concepción de la oferta y la aceptación como mecanismo de formación de los contratos es unánimemente reconocido tanto en los ordenamientos jurídicos nacionales como en los referentes internacionales. No obstante, existen características o tratamientos diferenciales en algunos aspectos relevantes que, a consideración del autor, merecen un especial análisis comparativo. A continuación, en consecuencia, se realiza un estudio sobre los elementos que se consideran de mayor relevancia en relación a la oferta.

\section{Contenido de la oferta - Falta de elementos esenciales}

La oferta entendida como la propuesta de celebrar un contrato que una persona dirige a una o varias personas determinadas (artículo 826 del CCoBol; artículo 845 del $\mathrm{CCoC}$ ). En igual sentido, Díez Picazo (2007, p. 330) expone que "la oferta es una declaración de voluntad en la que el oferente manifiesta su intención de alcanzar la formación de un contrato".

Ahora bien, en relación al contenido requerido en la oferta (también denominado determinación objetiva), los ordenamientos ju- rídicos nacionales en estudio coinciden en la necesidad de que la propuesta incorpore los elementos necesarios del negocio jurídico propuesto (artículo 826 del CCoBol; artículo 845 del CCoC.). Es así como la oferta se concibe como una proposición completa en la cual se contemplan la totalidad de elementos esenciales, de tal forma que con la aceptación simple y pura (artículo 145 del Código Civil de Ecuador, $\mathrm{CCoE})$ el contrato queda en el acto perfeccionado y surte todos sus efectos legales.

Sin embargo, puede suceder que la oferta no precise uno o más de los elementos sino que, por el contrario, posponga o reserve para una parte la fijación de los mismos; en otras palabras, puede que la propuesta de negocios sea indeterminada en algunos de sus aspectos (por ejemplo en una oferta mercantil de compraventa de un bien, en la cual no se determine específicamente el precio).

En estricto sentido, se podría sostener que un defecto como el descrito implicaría entender que la propuesta así concebida no es una verdadera oferta y, que por tanto, solo el acto posterior que incorpore la totalidad de elementos esenciales será tenido como tal (Cancino, 1979, p. 63). No cabe duda de que esta sería la posición conducente o aplicable de conformidad con las normas internas de los países miembros de la CAN (artículo 826 del CCoBol; artículo 845 del CCoC; artículo 145 del CCoE y artículo 1376 del CCoP). Más aún, si se examinan los pronunciamientos que sobre el particular ha proferido la Corte Suprema de Justicia $(1980,1995)$ de la República de Colombia, en los cuales expresamente señala que "si es incompleta (la oferta) es elemental que no puede producir efectos vinculantes, entendiéndose que los aspectos secundarios pueden ser especificados con posterioridad" (Oviedo, 2008a, pp. 36-39). Esta posición, a todas luces exegética de la norma, no se muestra armónica con los elementos de carácter internacional, en los cuales se propugna por la supervivencia o preservación de la voluntad y del negocio jurí- 
dico (Mendoza Ramírez, 2008, p. 13; Serrano Acitores, 2010) ${ }^{10}$, así como por cobijar con la mayor seguridad jurídica los diferentes actos o contratos.

Los Principios (artículo 2.2), por ejemplo, emplean una redacción amplia con relación a la oferta, en tanto exige que la propuesta sea "suficientemente precisa e indica la intención del oferente de quedar vinculado en caso de aceptación". Ha de entenderse que el concepto de precisión de la oferta hace referencia a la indicación de los elementos que hacen parte del contrato, cuando menos de una forma determinable, mediante la práctica o usos internacionalmente aceptados (Díez-Picazo, 2010, p. 33; Oviedo et al., 1999; Oviedo, 2001, 2004, 2007, 2008a, p. 38). Perales Viscasillas (1999) expone que la disposición de los Principios, tal y como se encuentra, evita disputas doctrinales (entre el civil law y el common law) en torno al concepto de elemento esencial y se muestra más acorde con la práctica comercial internacional.

Lo anterior encuentra sustento adicional en el artículo 2.1.14 de los Principios al disponer que "si las partes han tenido el propósito de celebrar un contrato, el hecho de que intencionalmente hayan dejado algún término sujeto a ulteriores negociaciones o a su determinación por un tercero no impedirá el perfeccionamiento del contrato".

No siendo ello suficiente en el ámbito internacional, la CISG, en su artículo 14.1, establece cuál ha de ser el contenido de la oferta, sin requerir que el mismo sea absolutamente completo sino que se limita a la imposición de un grado de suficiente precisión, permitiendo posibilidades distintas para determinarlos (Crucilaegui, 2005, p. 107), siempre que se tengan en cuenta criterios de razonabilidad (artículo 7.1), intención de los contratantes (artículo 8.1) y buena fe (artículo 8.2) (Oviedo, 2001, 2004, 2007, 2008a, p. 41; Perales Viscasillas, 1999, pp. 105-107).
Adicionalmente, ha de resaltarse que la misma Convención, en su artículo 55, prevé el mecanismo para suplir el vacío respecto del precio de la venta (contratos de precio abierto $\mathrm{u}$ open price contracts), acudiendo al generalmente cobrado en el momento de la celebración del contrato en el tráfico mercantil. En este sentido, si el artículo 920 del CCoC prevé la forma de determinación de un elemento esencial (el precio) en el evento de ser omitido en la formación de un contrato de compraventa, así como lo hace la CISG, ¿por qué razón no se admite la misma posibilidad para los demás contratos mercantiles? (Oviedo, 2008a, p. 39).

En conclusión, la redacción cerrada y la interpretación estricta de la delimitación objetiva de la oferta que se aplica en la actualidad en los países de la Comunidad Andina no es acorde o armónica con las corrientes internacionales. En este sentido, es evidente la necesidad de favorecer la seguridad jurídica del tráfico mercantil incorporando un concepto más amplio en el contenido de la oferta, omitiendo la sanción de inexistencia a las ofertas indeterminadas, esto es, en otras palabras, acogiendo como principal $\mathrm{y}$ verdadera fuente obligacional de la propuesta la intención del oferente de obligarse.

\section{Revocación de la oferta}

Una vez estudiados el contenido y el momento en el cual la oferta surte efectos jurídicos, es preciso analizar si en los diferentes elementos normativos de referencia le es reconocida fuerza obligatoria a la oferta. Oviedo et al. (1999) explica que no existe unanimidad en los diferentes sistemas jurídicos con relación a afirmar si esta compromete al oferente o, en otras palabras, si el destinatario puede obligar al oferente a mantener el contenido de la misma, así el primero ya no quiera o no esté en condiciones de obligarse conforme al mismo.

En términos generales, se han reconocido las siguientes corrientes teóricas: i) la denominada teoría francesa, según la cual la manifes- 
tación de voluntad no obliga al oferente, por tratarse de una declaración unilateral de la cual no se deriva responsabilidad alguna, ni es transmisible de forma alguna a los herederos; ii) la teoría alemana, que propugna por la obligatoriedad de la oferta, en tanto la considera como una declaración de la voluntad negocial y, por tanto, debe mantener su propuesta (no la puede revocar) y la misma se transmite a los herederos en caso de fallecimiento o incapacidad del creador, y finalmente, iii) la teoría ecléctica, que defiende la revocabilidad de la oferta, reconociendo excepciones, por ejemplo al fijar un plazo (Planiol \& Ripert, 1936, p. 189; Josserand, 1950, p. 41; Enneccerus, Kipp \& Wolff, 1955, artículo 145. p. 28, citado por Oviedo et al., 1999; Claro Solar, 1992, p. 83; Suescún Melo, 1996, p. 318; Oviedo et al., 1999). Así las cosas, al contrario de lo que pudiera pensarse, de manera inicial, los ordenamientos jurídicos de la Comunidad Andina se alejan mayoritariamente de la teoría francesa pura, orientándose hacía la teoría ecléctica y la alemana.

En Bolivia existe una dualidad de normas a estudiar. En primer término, el artículo 458 del CCBol establece la posibilidad de revocatoria de la oferta mientras la aceptación no sea de conocimiento del oferente (teoría francesa), sin embargo, en el segundo inciso del mismo artículo modula la posición inicial al reconocer que si el destinatario, sin tener noticia de la revocación del aceptante, da inicio de buena fe en la ejecución del negocio, "se hace beneficiario de la indemnización que debe reconocerle el oferente por los gastos y pérdidas sufridas" (teoría ecléctica).

Desde otra perspectiva, el artículo 826 del CCoBol define claramente que la oferta, una vez comunicada al destinatario, no podrá ser revocada so pena de indemnizar los perjuicios que con ello se causen al destinatario (teoría alemana). Con lo cual, del estudio sistemático, es claro que el ordenamiento boliviano no se inclina por la teoría francesa, sino que acoge una tendencia variada para reconocer la responsabi- lidad del oferente de pagar perjuicios en caso de revocación de la propuesta.

En Colombia, el artículo 846 del CCoC, y en Perú, el artículo 1382 del $\mathrm{CCP}^{11}$, disponen de forma tajante la obligatoriedad de la oferta $y$, en consecuencia, la irrevocabilidad de la misma. En otras palabras, en estos ordenamientos la manifestación de voluntad (oferta) vincula a quien la formula, pues si se retracta de la misma debe indemnizar los perjuicios que se causen, aun si este no ha expresado su aceptación y, adicionalmente, en caso de que la aceptación se hubiera expedido en debida forma, el contrato se hubiera formado y el destinatario sería titular de las acciones y derechos contractuales por incumplimiento.

Finalmente, en el caso del Ecuador, el artículo 143 del CCoE parece, en primera medida, acoger la tesis ecléctica al disponer que el proponente puede arrepentirse en el tiempo que medie entre el envío de la propuesta y la aceptación, con la excepción de que la oferta se hubiere comprometido a esperar contestación o a no disponer del objeto del contrato, caso en el cual deberá esperar el plazo establecido. Sin embargo, si se estudia la norma en cita al amparo del artículo 144 del mismo estatuto, es evidente que se acoge de forma clara la teoría alemana, al aclarar que la retractación impone al proponente la obligación de indemnizar los gastos y los daños y perjuicios que hubiere sufrido el destinatario y que solo podrá exonerarse cumpliendo el contrato propuesto.

Ahora bien, en el marco de los elementos internacionales en estudio, tanto los Principios, en su artículo 2.4, como la convención, en el artículo 16, evocan una clara teoría ecléctica en la cual la oferta es revocable siempre y cuando la comunicación de la misma llega al destinatario antes de la emisión de la aceptación. Estableciendo, en todo caso, tres circunstancias de irrevocabilidad a saber: i) cuando la oferta indica que es irrevocable, ii) cuando se señale un plazo determinado para la aceptación y iii) cuando el 
destinatario pueda razonablemente considerar la oferta como irrevocable y procede conforme a ello (Crucilaegui, 2005, pp. 112-114; Oviedo et al., 1999), esta última sustentada en la legítima confianza y expectativa que determinados comportamientos generan.

Es de anotar que, como en los casos citados de los principios y la CISG, la revocación de la oferta requiere de su comunicación para alcanzar efectividad y que esta llegue al destinatario antes de que se emita la aceptación. En este punto se concluye que la obligatoriedad y subsecuente irrevocabilidad de la oferta es un elemento más o menos armónico en los ordenamientos de la Comunidad Andina y frente a los instrumentos de carácter internacional en estudio. En este sentido, la similitud normativa es un elemento claramente favorable en la vocación de internacionalidad de los sistemas jurídicos latinoamericanos.

\section{Aceptación}

Entendemos por aceptación la manifestación de voluntad proveniente del destinatario de una oferta por medio de la cual se adhiere a los términos de la misma, generando, como efecto jurídico, el perfeccionamiento del contrato propuesto (Galán Barrera, 2003; Oviedo, 1999; Suescún Melo, 1996, p. 352; Ospina Fernández, 1994, p. 162). En este sentido, es generalmente aceptado, en los ordenamientos nacionales y en los elementos de contexto internacional, que con la aceptación se concreta el acuerdo de las voluntades negociales de los co-contratantes. No obstante lo anterior, el alcance y el momento en el cual ello sucede difieren en los diferentes ordenamientos de referencia, como se evidencia en el análisis realizado en el presente acápite.

\section{Modos de la aceptación}

En términos generales, la aceptación puede manifestarse de forma expresa o tácita. $\mathrm{La}$ primera, es aquella en la cual el destinatario realiza una declaración o expresión de voluntad directa de conformidad con los términos de la oferta. La segunda, por el contrario, no parte de una comunicación al oferente, sino que se sustenta en la realización del destinatario de actos que denoten el cumplimiento del contrato y de los cuales se infiere el asentimiento de la oferta.

Es precisamente esta segunda forma de manifestar la aceptación de la oferta la que merece un estudio más profundo. En este sentido, es preciso advertir que la mayoría de los ordenamientos de la CAN reconocen, normativamente, la posibilidad de que el contrato se forme mediante la aceptación tácita del beneficiario, lo cual, en principio, es una posición favorable a la finalidad de armonización ${ }^{12}$. Sin embargo, las condiciones en las cuales se reconocen difieren en algunos sentidos.

En particular, Bolivia y Perú (inc. 2 del artículo 455 del CCBol; artículo 1380 CCP; artículo 457 CCOP) restringen la procedencia de la aceptación tácita cuando ello sea procedente "de acuerdo a los usos o a la naturaleza del negocio o por solicitud del oferente, la prestación ha de ejecutarse sin respuesta previa". En este sentido, para los ordenamientos en estudio, en principio, no todos los contratos son susceptibles de ser aceptados de forma tácita, salvo que se pueda demostrar alguno de los eventos o condiciones anteriores.

Asimismo en la normatividad de Bolivia y Perú se contempla, en todo caso, la obligación al destinatario de dar aviso del inicio de la ejecución del contrato, so pena de indemnizar los perjuicios ${ }^{13}$. Es evidente que en estos casos se restringe en especial forma la aceptación tácita de la oferta, dando especial preponderancia a la aceptación expresa.

Desde la perspectiva del derecho colombiano, el artículo 854 del CCoC expone de manera genérica que la aceptación tácita produce los mismo efectos que la expresa, bajo el supuesto que el oferente tenga conocimiento de 
los hechos o actos constitutivos de la aceptación de manera oportuna (esto es, dentro de los plazos fijados para cada una de las modalidades de la oferta, es decir, si es verbal, escrita o con plazo acordado). En este sentido, la norma colombiana parece ser más amplia, en tanto no restringe la aplicación de la aceptación tácita al cumplimiento de presupuesto alguno, como se mencionó en los casos boliviano y peruano.

De igual forma, se muestra más abierta la redacción de la norma colombiana frente a las estudiadas en el sentido que no impone la carga al destinatario de dar aviso de los hechos, sino que se limita a exigir que el oferente tenga conocimiento oportuno. Lo anterior se muestra relevante en el entendido que el conocimiento oportuno del oferente puede derivarse de una fuente diferente al aviso del aceptante, con lo cual se impide que, eventualmente, el oferente se ampare en el no recibo del aviso de notificación como excusa de su incumplimiento.

Ahora bien, en el entorno internacional tanto los Principios (artículo 2.1.6) como la CISG (artículo 18.3) concuerdan en reconocer que, si "en virtud de la oferta o de las prácticas que las partes hayan establecido entre ellas o de los usos", se infiere que el destinatario puede manifestar su asentimiento mediante actos de ejecución del contrato, no se negarán efectos a dicha situación siempre y cuando los mismo sean oportunos, aun sin notificación al oferente.

Para Crucilaegui (2005, p. 116) la regla general para la aceptación, expresa o tácita, es ser comunicada al oferente, pues de otro modo no producirá efectos. Es así como el autor destaca que lo dispuesto específicamente en el artículo 18.3 de la Convención, en relación con la no notificación al oferente, ha de tratarse como una excepción con interpretación restrictiva, no aplicable a todas las situaciones negociales.

Desde otra perspectiva, casi todos los ordenamientos nacionales, así como los referentes internacionales en estudio, coinciden, como regla general, en negar efectos jurídicos a la simple inacción o silencio del destinatario como aceptación. No obstante, se identifican situaciones excepcionales como las siguientes:

- En Bolivia, el artículo 460 del CCBol expone que el silencio puede constituir una manifestación de voluntad si los usos o las circunstancias lo autorizan y si el contrato o la ley no expresan lo contrario.

- Colombia, el artículo 2151 del CCC presume que el silencio es aceptación en el caso del contrato de mandato frente a las personas que por su profesión u oficio se encargan de negocios ajenos.

- Para Perales Viscasillas (1999, p. 118), en el marco de los Principios el silencio puede ser válido como aceptación si se deriva de un acuerdo expreso de las partes (artículo 1.1), de los usos o prácticas establecidas (artículo 1.8), o cuando otros preceptos lo reconozcan de esa manera (artículo 2.22 (2) y artículo 2.9 (1)) (Oviedo Albán et al., 1999).

- Crucilaegui (2005, p. 116) expone cómo en algunos casos respecto de la CISG se ha interpretado excesivamente el silencio como aceptación, a la luz del principio de buena fe, de los usos del sector o de las prácticas de las partes, a tal punto que el autor sugiere al destinatario contestar, así sea en forma negativa o dilatoria, para no dejar su silencio al arbitrio del juez.

A partir de lo anterior, se evidencia que el reconocimiento de la aceptación tácita es un elemento más o menos armónico en los ordenamientos de la Comunidad Andina y frente a los instrumentos de carácter internacional en estudio, lo que se muestra como un elemento 
claramente favorable en la vocación de internacionalidad de los sistemas jurídicos latinoamericanos.

\section{Contenido de la aceptación}

La doctrina tradicional implementada tanto en el common law y en el derecho continental respecto al contenido de la aceptación en la formación del contrato se guía por la denominada regla del espejo (mirror image rule), según la cual la aceptación debe ser un reflejo de la oferta en el espejo, esto es sin adicionar, modificar, matizar o exceptuar condición alguna, so pena de entenderse rechazada la oferta inicial y de entender esta, la aceptación modificativa, como una nueva oferta (contraoferta) (Perales Viscasillas, 2001).

Es precisamente esta concepción teórica la que es incorporada, de forma estricta, en los ordenamientos jurídicos de la Comunidad Andina. Lo anterior es evidente si se revisan los artículos 456 del CCBol, 855 del CCoC, 145 del CCoE, 1375 y 1376 del CCP, según los cuales la aceptación condicionada, en cualquier sentido, es considerada como una nueva propuesta. Sin embargo, esta tradicional regla ha venido siendo matizada o restringida en los diferentes escenarios jurídicos. En el ámbito angloamericano, se restringe su aplicación al common law tradicional en el evento en que no se disponga de ningún tipo de intercambio de formularios pues, en caso contrario, será aplicable la sección 2-207 del Uniform Comercial Code (UCC), según el cual una expresión definitiva de aceptación o una confirmación escrita enviada en un término razonable opera como una aprobación aunque incluya términos adicionales o diferentes a los de la oferta inicial, a menos que en la aceptación se exprese la condición de que se admitan las nuevas disposiciones ${ }^{14}$.

Desde otra perspectiva, los artículos 2.1.11 de los Principios y 19 de la Convención, aunque en primera medida acogen la teoría del espejo, aclaran a renglón seguido que, no obs- tante lo anterior, también es tenida como una aceptación la respuesta que contenga estipulaciones adicionales o diferentes, siempre y cuando se cumplan las siguientes condiciones: i) que la respuesta pretenda ser una aceptación, esto es que el destinatario manifiesta su intención de quedar vinculado, ii) que las modificaciones no alteren sustancialmente los términos de la oferta inicial y iii) que las alteraciones no sean objetadas, sin demora injustificada, por el oferente.

Para Perales Viscasillas (1999), este tipo de disposiciones se muestra acorde con las corrientes normativas internacionales, la compleja realidad comercial y se aleja de la rigidez de la regla del espejo ${ }^{15}$. Con relación a los Principios, la redacción según la cual es necesario que las modificaciones no sean sustanciales se muestra abierta.

En este sentido, Oviedo Albán (1999) aclara que no es posible clasificar las modificaciones sustanciales de forma abstracta, sino que por el contrario ha de analizarse cada caso en concreto, es decir, "dependiendo de cuáles sean los elementos del contrato cuya celebración se esté ofreciendo, así como si son elementos adicionales o diferentes a los regularmente utilizados en la rama comercial de que se trate". En todo caso, es de resaltar que toda interpretación del contrato ha de cobijarse bajo los principios de respeto a la voluntad de las partes, buena fe contractual y lealtad negocial.

Desde la perspectiva de la CISG, en el artículo 19.3 se clarifica el contenido de la clasificación de esencial a la modificación, al indicar que se consideran como tal aquellas relativas al precio, al pago, a la calidad y la cantidad de las mercaderías, al lugar y fecha de la entrega, al grado de responsabilidad de una parte con respecto a la otra o a la solución de las controversias.

De acuerdo a lo anterior, es evidente que existe total coincidencia entre los ordenamientos de la Comunidad Andina en lo relativo al 
contenido de la aceptación. Sin embargo, la teoría incorporada en los mismos se muestra disímil de la tendencia internacional y por tanto disonante de los instrumentos en estudio. Así las cosas, se considera conveniente resolver la disparidad normativa a este respecto, de tal forma que los ordenamientos nacionales se armonicen al incluir, con vocación de internacionalidad, las corrientes vigentes con relación a la flexibilización de la regla del espejo.

\section{La formación del contrato electrónico}

Como se mencionó anteriormente en este documento, todos los ordenamientos jurídicos de la Comunidad Andina, así como los diferentes elementos normativos de carácter internacional, contemplan el principio de consensualidad y por tanto la posibilidad de que los comerciantes expresen su voluntad de contratar y de obligarse por cualquier medio inequívocamente dirigido a tal fin, lo cual, sin duda alguna, incluye los medios electrónicos, salvo que una norma imperativa exija una solemnidad especial como requisito esencial para la validez del contrato, en cuyo caso este último no se perfecciona sino hasta cuando la mencionada solemnidad sea satisfecha.

En igual sentido, Díez Picazo (1993, p. 248) sostiene que el concepto de forma del contrato se identifica con todo medio que le pueda servir de vehículo a su exteriorización. Así las cosas, los actos, manifestaciones, acuerdos o contratos surten efectos jurídicos cualquiera que sea la forma o el medio a través del cual se hayan celebrado, con lo cual se logra, de comienzo, un claro reconocimiento a la formación y a la existencia del contrato electrónico.

Sin embargo, este no es el único fundamento normativo de las manifestaciones electrónicas de voluntad. Desde la Ley Modelo sobre Comercio Electrónico de la Comisión naciones Unidas para el Derecho Mercantil Internacional se estableció, como principio, el reconocimiento de la validez de las comunicaciones electró- nicas, el cual ha sido, a su turno, incorporado en la mayoría de los ordenamientos jurídicos de la Comunidad Andina. El principio en estudio se puede definir como la prohibición de negar, eficacia obligatoria y probatoria, cualquier tipo de información en forma de mensaje de datos por el solo hecho de tratarse de su forma.

Sin embargo, esto no es ajeno a lo ya estudiado respecto a la consensualidad, en el sentido que el alcance del reconocimiento de los mensajes de datos procede siempre y cuando una norma de carácter imperativo no contemple una solemnidad sustancial específica.

En los diferentes ordenamientos jurídicos de la CAN se dispone de manera expresa que no se negarán efectos jurídicos, validez o fuerza obligatoria a todo tipo de información por la sola razón de que esté en forma de mensaje de datos. Como evidencia de lo anterior, es posible citar el artículo 1 del proyecto de ley 080/2007 (Bolivia), artículo 5 de la Ley 527 de 1999 (Colombia), artículo 2 de la Ley n. ${ }^{\circ} 67$ de 2002 (Ecuador) y el artículo 1 de la Ley 27291 de 2000 (Perú).

Ahora bien, el principio de equivalencia funcional es el principal instrumento para la regulación de los actos y negocios jurídicos realizados con ayuda de las TIC, como un criterio internacional de interpretación de las distintas normas del ordenamiento jurídico que regulan el valor probatorio de un escrito, un documento firmado, un original o la conservación de un documento, entre otras, pueda equipararse a los mensajes de datos (Umaña Chaux, 2005).

Así pues, es posible definir el principio de equivalencia funcional como la paridad de las instituciones jurídicas del mundo material en el contexto digital, una vez se analiza el cumplimiento de las finalidades y objetivos de la figura en el mundo digital. (Rincón Cárdenas, 2006; Remolina Angarita, 2006; Olivera, 2008; Espinosa Quintero, 2008). Al respecto, se destaca que en los diferentes ordenamientos jurídicos 
de la CAN se incorpora la equivalencia funcional, para lo cual es posible citar los artículos 4 b) y c), 7, 8 y 9 del proyecto de ley $080 / 2007$ (Bolivia); artículos 6, 7 y 8 de la Ley 527 de 1999 (Colombia); artículos 6 y 7 de la Ley n. ${ }^{\circ}$ 67 de 2002 (Ecuador) y los artículos 2 y 3 de la Ley 27291 de 2000 (Perú).

A partir de lo anterior, se evidencia que el reconocimiento de los principios fundamentales de la contratación electrónica es un elemento armónico en los diferentes ordenamientos de la Comunidad Andina, así como se muestra claramente acorde con los instrumentos de carácter internacional en estudio, lo cual se considera un elemento claramente favorable en la vocación de internacionalidad de los sistemas jurídicos latinoamericanos. En todo caso, y además teniendo en cuenta que Bolivia, a la fecha, todavía no ha culminado su proceso de normatización del comercio y la contratación electrónica, se considera de especial valía e importancia el desarrollo de una legislación armónica a nivel andino, que a modo de propuesta regional permita alinear los ordenamientos jurídicos nacionales con las tendencias jurídicas internacionales.

\section{Conclusiones}

El derecho en su función reguladora de las relaciones sociales, encaminado al mantenimiento de la convivencia al interior de la sociedad, es elemento fundamental en el desarrollo de la denominada Sociedad de la Información, ya que así como no se concibe una vida social sin derecho, no es aceptable el desarrollo de las nuevas tecnologías sin regulación.

Así las cosas, se destaca que la mayoría de los países de la Comunidad Andina han incorporado a sus ordenamientos jurídicos, normatividades o regulaciones que responden a necesidades y concepciones particulares, en últimas, que atienden una concepción nacional. En el ámbito comunitario se evidencia, y así se ha declarado, la necesidad de armonizar o uni- ficar la normativa respecto del entorno digital, de establecer principios y medidas dentro de las organizaciones regionales existentes, en el caso latinoamericano la Comunidad Andina (CAN), que permitan dotar de seguridad jurídica a los actores de las relaciones electrónicas.

Sin embargo, siguiendo a Garro (1993), la armonización o unificación del derecho privado en América Latina no puede centrarse en una posición tradicional, esto es, que se sustente únicamente en la realidad de los países de la región, sino que, por el contrario, el proceso debe guiarse hacia la solución de genuinas diferencias jurídicas con otros ordenamientos con los cuales se mantienen constantes relaciones comerciales, así como con los elementos e instrumentos internacionales de referencia para nuestros sistemas.

Es así como el presente estudio evidenció la situación actual de la normatividad de los países miembros de la CAN respecto de algunos aspectos relativos a la formación del contrato electrónico, desde una perspectiva regional e internacional, al contrastarlos con las soluciones que, en las mismas materias, han dado, principalmente, los Principios Unidroit y la Convención de Viena de 1980. La comparación planteada se muestra, adicionalmente, apropiada si se tiene en cuenta que en el contexto europeo, Perales Viscasillas (2009) resalta que, en términos generales, las disposiciones relativas a la formación del contrato del Marco Común de Referencia se inspiran fuertemente en lo incorporado en los Principios y en la CISG.

Es de resaltar que la mayoría de los países ha incluido en sus ordenamientos el reconocimiento de los principios fundamentales (validez de las manifestaciones realizadas por medios electrónicos y equivalencia funcional) de la contratación electrónica, así como un unánime reconocimiento del principio de consensualidad, elementos que son claramente armónicos en los diferentes ordenamientos de la Comunidad Andina en relación con los instrumentos 
internacionales y que por tanto facilitan o soportarían una eventual unificación.

A su turno, la obligatoriedad y subsecuente irrevocabilidad de la oferta, así como el reconocimiento de la aceptación tácita de la misma, se muestran como otros elementos favorables en la vocación de internacionalidad de los sistemas jurídicos.

En este sentido, se considera de especial importancia el desarrollo de una legislación armónica a nivel andino, que a modo de propuesta regional permita alinear los ordenamientos jurídicos nacionales con las tendencias jurídicas internacionales, más aun si se recuerda que Bolivia todavía no ha culminado su proceso de normatización del comercio y la contratación electrónica.

No obstante lo anterior, son varias las materias, en relación con la formación del contrato, las que presentan disparidades ya sea entre los mismos ordenamientos andinos o entre estos y los referentes internacionales. En particular, y contraria a su naturaleza y finalidad, las normativas mercantiles de los países se muestran rígidas y alejadas de la realidad del tráfico negocial. Así pues, son variadas las dificultades o elementos dispares, tales como:

- La cerrada redacción e interpretación de la delimitación objetiva de la oferta en los países de la CAN que sancionan con inexistencia a las ofertas indeterminadas, mientras que los elementos internacionales acogen como principal y verdadera fuente obligacional de la propuesta, la intención del oferente de obligarse.

- El momento en el cual la oferta cobra eficacia jurídica, pues la mayoritaria incorporación de la teoría de la expedición difiere de los instrumentos de carácter internacional estudiados, los cuales optan por acoger la teoría de la recepción.
- Respecto al momento en el cual la aceptación cobra eficacia jurídica, se ha de tener en cuenta en los ordenamientos colombiano y peruano, son los únicos, de los estudiados, que todavía acogen la teoría de la expedición de la oferta como momento de formación del contrato.

- La total coincidencia entre los ordenamientos de la Comunidad Andina en lo relativo al contenido de la aceptación (teoría del espejo) difiere de la tendencia internacional, orientada a la flexibilización y al reconocimiento como contrato del acuerdo sobre los elementos esenciales.

En todos los casos se ha de propiciar la armonización de los ordenamientos nacionales frente a los referentes internacionales, siempre teniendo en cuenta que la vocación de ayuda y apoyo del tráfico mercantil han de inspirar a la normatividad mercantil en aras de dar seguridad jurídica a las diferentes situaciones negociales.

Así pues, se encontraron elementos de notable similitud que podrían servir de soporte a una propuesta de unificación a nivel de la Comunidad Andina. Sin embargo, también se evidencian grandes diferencias y disparidades en algunos aspectos relativos a la formación del contrato electrónico, que, en últimas, permiten apreciar la inexistencia de una estrategia armonizadora clara a nivel regional, así como los problemas para la integración normativa en la materia.

\section{Notas}

${ }^{1}$ Para Galgano (2005, pp. 105 - 112) las concepciones clásicas del derecho no ubican al contrato entre las fuentes normativas. Sin embargo, continuar concibiendo el contrato como mera aplicación del derecho, y no como una fuente del nuevo derecho, implica no comprender de qué modo cambia el derecho de nuestro 
tiempo. "Este es el caso de los códigos de comportamiento, o deontológicos, redactados por asociaciones de categorías o por sociedades de gestión de mercados reglamentados, receptados por el nuevo artículo 2387 del código civil (italiano)”. El artículo 2387 del nuevo Código Civil Italiano, en su parte pertinente, expresa que los estatutos (de la sociedad anónima) podrán formular las exigencias de honorabilidad, profesionalidad y la independencia del administrador para la celebración de contratos, también, con referencia a estos requisitos, podrá resguardarse en los códigos de conducta elaborados por asociaciones comerciales o por las sociedades de gestión de los mercados regulados. "Lo statuto può subordinare l'assunzione della carica di amministratore al possesso di speciali requisiti di onorabilità, professionalità ed indipendenza, anche con riferimento ai requisiti al riguardo previsti da codici di comportamento redatti da associazioni di categoria o da società di gestione di mercati regolamentati”.

${ }^{2}$ En este mismo sentido, Gutiérrez Gómez (2005, p. 33) destaca, como un escenario adicional a nivel internacional para el debate sobre el marco jurídico del comercio electrónico, los dos informes de Recomendaciones para los Ministros del Área de Libre Comercio para las Américas (ALCA) del Comité Conjunto de Expertos del Gobierno y del Sector Privado sobre Comercio Electrónico, en los cuales se han abordado temas trasversales tales como el fortalecimiento de la infraestructura, el aumento de la participación, la aclaración de las reglas del mercado, sistemas de pago en línea, certificación y autenticación, propiedad intelectual, entre otros. Asimismo la agenda del Comité tiene por delante el estudio de temas como la brecha digital, la protección del consumidor y el gobierno electrónico.

${ }^{3}$ Sobre la seguridad jurídica se han planteado diversas definiciones que se encuentran altamente ligadas a la línea de pensamiento de cada autor o filosofo del Derecho. Por ejemplo, para Radbruch (1947, p. 40) la seguridad jurídi- ca es conferida por el Derecho mismo, al garantizar la vida y bienes de las personas, siempre que se sujete a cuatro condiciones a saber: i) que el Derecho se halle estatuido en leyes; ii) que el Derecho se base en hechos y que no se remita a los juicios de valor del juez; iii) que los hechos en que se basa el Derecho sean practicables, y iv) finalmente, el Derecho no debe hallarse expuesto a cambios demasiados frecuentes. Es evidente que la definición de "seguridad jurídica", formulada por el autor reseñado, está relacionada con una tendencia marcadamente formalista del Derecho. Para Kelsen (1995, p. 260), el principio de seguridad jurídica deriva en la previsibilidad de las decisiones de los tribunales, que sirve a los destinatarios del derecho para orientar su comportamiento. En este mismo sentido, Corte Constitucional (1995, A.V. C-194, Mag. Cifuentes, Martínez y Naranjo). Ahora bien, para la Corte Constitucional colombiana el principio de seguridad jurídica implica, entre otras formas o efectos, la confianza del conglomerado social en lo resuelto, sin que surjan nuevas acciones que provoquen la indefinida reanudación de procesos. Ver: Corte Constitucional (1992, C-543; 2001, SU-014).

${ }^{4}$ En el presente artículo se tendrá como referente internacional, y en especial como instrumento integrante de la lex mercatoria a los Principios Unidroit sobre contratos internacionales. Sin embargo, vale la pena destacar que existen en la doctrina opiniones encontradas sobre la aplicación inmediata de los Principios cuando las partes acuerdan someter su relación jurídica a la lex mecatoria. Al respecto, Perales Viscasillas (1999) expone que el tercer inciso del preámbulo de los principios, a diferencia de los anteriores, utiliza la expresión "pueden aplicarse" y no "deberán aplicarse", con lo cual se está realizando una recomendación a los operadores jurídicos, mas no imponiendo su empleo como parte de la lex mercatoria.

${ }^{5}$ El Instituto Internacional para la Unificación del Derecho Privado (Unidroit) es una organización intergubernamental independiente 
con sede en Roma, creada con el objetivo de estudiar las necesidades y métodos para modernizar, armonizar y coordinar las legislaciones privadas y, en particular, el derecho comercial entre los Estados y grupos de Estados, con el fin de lograr una normatividad de derecho privado uniforme (Oviedo, 2008b, p. 44 y ss).

${ }^{6}$ Se destaca que de los países de la Comunidad Andina solo Bolivia, a la fecha, no ha ratificado el texto de la CISG. Así pues, las normas aplicables a la compraventa internacional de mercaderías contenidas en la Convención son aplicables en Colombia (entrada en vigor: 2002), Ecuador (entrada en vigor: 1993) y Perú (entrada en vigor: 2000) (Cnudmi, 2011).

${ }^{7}$ No obstante lo anterior, en el artículo 9.2 la DCE aclara que, en todo caso, los Estados parte podrán disponer que la libertad de forma antes mencionada no se aplique a unas categorías específicas de contratos, por ejemplo, relacionadas con derechos inmobiliarios, contratos en los que intervengan autoridades públicas o relacionadas con asuntos de familia o de sucesiones.

${ }^{8}$ De igual forma se muestran como destacables los siguientes pronunciamientos judiciales o administrativos, en los cuales se reconoce la aplicación del principio de consensualidad: Superintendente Tributario General (2004), Corte Suprema de Justicia (1981), Corte Suprema de Justicia (2000a, 2000b, 2004a, 2004b).

${ }^{9}$ En Colombia se consideran como normas excepcionales en relación con el principio de consensualidad, por ejemplo, los artículos 119 (contrato promesa de contrato de sociedad comercial); 184 (poder de los socios para representación en las reuniones de junta de socios o asambleas de accionistas); 836 (poder para celebrar negocio jurídico sujeto a escritura pública); 526 (enajenación de establecimiento de comercio) del CCC, en las cuales se exigen solemnidades adicionales a la expresión de la voluntad, tales como la necesidad de un escrito y, en algu- nos casos, la autenticación de la firma ante un funcionario que administre la fe pública.

${ }^{10}$ Entiéndase por el principio de preservación del negocio jurídico (favor negotti) la vocación de un ordenamiento jurídico de "salvar", hasta donde sea posible, la voluntad expresada por las partes al celebrar cualquier negocio jurídico. Para Gómez Vásquez (2006, p. 9) aunque en realidad la naturaleza de principio del favor negotti es discutible, sí es evidente la tendencia más o menos general hacia la efectividad o eficacia de la disposición negocial, salvo que la misma supere en gran medida los límites éticos y de corrección fijados por la estructura legal del contrato.

${ }^{11} \mathrm{Ha}$ de recordarse en todo caso, como se explicó con anterioridad, que la eficacia de la oferta en el ordenamiento jurídico peruano se deriva de la recepción del destinatario. Es así como, según las voces del artículo 1384 del CCP, la oferta deja de ser obligatoria si antes o simultáneamente con su recepción llega a conocimiento del destinatario la declaración del oferente en el sentido de que puede revocarla en cualquier momento antes de su aceptación.

${ }^{12}$ Los códigos civil y de comercio de la República del Ecuador no hacen referencia alguna a la aceptación tácita, por el contrario, la redacción del artículo 145 del CCoE parece no dar cabida a la misma, al expresar que: "Dada la contestación, si en ella se aprobare pura y simplemente la propuesta, el contrato queda en el acto perfeccionado y surte todos sus efectos legales, a no ser que antes de darse la respuesta ocurra la retractación, muerte o incapacidad legal del proponente(...)".

${ }^{13}$ En este punto difieren un poco los dos ordenamientos, pues la normatividad boliviana exige un aviso inmediato, mientras que la norma peruana hace referencia a un aviso pronto al oferente.

${ }^{14}$ De manera textual, la sección 2-207 (1) del UCC expresa: "A definite and seasonable expression of acceptance or a written confir- 
mation which is sent within a reasonable time operates as an acceptance even though it states terms additional to or different from those offered or agreed upon, unless acceptance is expressly made conditional on assent to the additional or different terms". Sobre la formación y el contenido del contrato al amparo de las reglas contenidas en la sección 2-207 del UCC, vid. Perales Viscasillas (1998).

${ }^{15}$ En igual sentido, Aymerich Ojea (2003, p. 148) expresa que, en virtud de la CISG, tanto en los sistemas civilistas como en los angloamericanos los acuerdos incompletos se encuentran en fase de evolución a partir de un paso gradual de la total conformidad entre oferta y demanda al reconocimiento como contrato del acuerdo sobre los elementos esenciales. Para Orduña Moreno (2002, p. 443), citando a Díez Picazo, la admisión de términos adicionales o diferentes en la aceptación de la oferta, como elemento flexibilizador de la regla del espejo, entre otras previsiones, favorece la perfección del contrato $\mathrm{y}$, por tanto, la existencia de la relación negocial atenuando la exigencia de una perfecta o acabada concordancia de voluntades, tendencia esta auspiciada por el principio favor contractus.

\section{Referencias}

Alvarado Herrera, L., Clavero Ternero, M., Illescas Ortiz, R., Madrid Parra, A., Morán Bovio, D. (coord.)., Pendón Meléndez, M. A., Perales Viscasillas, P; Pulido Begines, J. L. (1999). Comentario a los principios Unidroit para los contratos del comercio internacional. Madrid: Aranzadi.

Aymerich Ojea, I. (2003). Lecciones de derecho comparado. Madrid: Universitat Jaume I. Recuperado de http://books.google.com. co/books?id=p7eElwNFQzQC\&hl=es\&s ource $=$ gbs_navlinks_s

Bulla Quintana, R. (2008). Nociones preliminares de lógica. Bogotá: Universidad Sergio Arboleda.
Calderón Rodríguez, C. (2000). El comercio electrónico: nueva lex mercatoria del comercio internacional. Revista de Derecho Informático Alfa Redi. Recuperado de http://www. alfa-redi.org/rdi-articulo.shtml? $\mathrm{x}=553$

Cancino, F. (1979). Estudios de derecho privado. Bogotá: Temis.

Cibertribunal Peruano, (2008). Recuperado de http://www.cibertribunalperuano.org/ portalcibertribunal/Principal.aspx

Claro Solar, L. (1992). Explicaciones de derecho civil chileno y comparado. Tomo undécimo. De las obligaciones II. Bogotá: Editorial jurídica de Chile: Editorial Temis S. A.

Comisión de Naciones Unidas para el Derecho Mercantil Internacional (Cnudmi) (1996). Guía para la incorporación de la Ley Modelo de la CNUDMI sobre Comercio Electrónico. Recuperado de http:// www.uncitral.org/pdf/spanish/texts/ electcom/05-89453_S_Ebook.pdf

Comisión de las Naciones Unidas para el Derecho Mercantil Internacional (Cnudmi) (2011). Situación actual. Convención de las Naciones Unidas sobre los contratos de compraventa internacional de mercaderías. Recuperado de http://www.uncitral.org/uncitral/es/uncitral_texts/sale_ goods/1980CISG_status.html

Comunidad Andina de Naciones (2000). Documento de Lima sobre la Sociedad Global de la Información. Comunidad Andina de Naciones.

Corte Constitucional (1992). Sentencia C-543. M. P. José Gregorio Hernández Galindo.

Corte Constitucional (1995). Aclaración de Voto Sentencia C-194. Mags. Cifuentes Muñoz, E.; Martínez Caballero, A. y Naranjo Mesa, V. 
Corte Constitucional (2000). Sentencia C-529. M.P. Antonio Barrera Carbonell.

Corte Constitucional (2001). Sentencia SU-014. M. P. Martha Victoria Sáchica Méndez.

Corte Suprema de Justicia (1980). Sentencia de 16 de octubre. M. P. Ricardo Uribe Holguín.

Corte Suprema de Justicia (1981). Sentencia del 13 de noviembre. Gaceta Judicial n. ${ }^{\circ}$ 2407, pp. 610-623. Colombia.

Corte Suprema de Justicia (1995). Sentencia de 8 de marzo. M. P. Pedro Lafont Pianetta.

Corte Suprema de Justicia (2000a). Sentencia del 12 de septiembre. Exp. 5397. Colombia.

Corte Suprema de Justicia (2000b). Sala Laboral. Sentencia n. ${ }^{\circ} 2632$ del 15 de febrero. Perú.

Corte Suprema de Justicia (2004a). Primera Sala de lo civil y mercantil. Sentencia n. $^{\circ} 82 \mathrm{del}$ 26 de abril. Exp. n. ${ }^{\circ}$ 179-2003. Ecuador.

Corte Suprema de Justicia (2004b). Sala Laboral. Sentencia n. ${ }^{\circ} 5132004$ del 23 de junio. Perú.

Crucilaegui, J. S. J. (2005). Contrato de compraventa internacional de mercaderías. Convención de viena de 1980 y otros textos complementarios. Navarra (España): Thomson: Civitas.

Cumbre Mundial sobre la Sociedad de la Información. (2004). Declaración de principios. Recuperado de http://www.itu.int/ wsis/geneva/index-es.html

De Miguel Asensio, P. (2005). Derecho del comercio electrónico. México: Porrúa.

Del Bosch Portolés, M., Unceta Laborda, M. (2005). Los principios Unidroit como lex contractus. En Cuestiones actuales del derecho mercantil. Madrid: Colex.

Díez Picazo, L. (1993). Fundamentos del derecho civil patrimonial (Vol. I. $4^{\mathrm{a}}$ ed.). Madrid: Civitas.

Díez-Picazo, L. (2007). Fundamentos del derecho civil patrimonial. I. Introducción. Teoría del Contrato. Madrid: Thomson Civitas.

Díez-Picazo, L. (2010). La formación del contrato de compraventa internacional de mercaderías. Oviedo Albán, J. (cord.). Obligaciones y contratos en el derecho contemporáneo. Bogotá: Universidad de la Sabana: Dike.

Enneccerus, L., Kipp, T., \& Wolff, M. (1955). Apéndice a la obra Tratado de Derecho Civil. Barcelona: Bosch Casa Editorial.

Espinosa Quintero, L. (2007). Incidencia de los medios electrónicos en el campo contractual colombiano. Revista Civilizar. Universidad Sergio Arboleda. Recuperado de http://www.usa.edu.co/civilizar/incidencia_medios.htm

Espinosa Quintero, L. (2008). La adaptación del ordenamiento jurídico colombiano a la contratación por medios electrónicos: un análisis desde la perspectiva del derecho comparado. Bogotá: Universidad Sergio Arboleda.

Fernández Rozas, J. C. (2000). El derecho del comercio internacional en el contorno de la globalización. Revista del Colegio de Notarios del Estado de México.

Fernández Rozas, J. C. (2004). Ius mercatorum. Autoregulación y unificación del derecho de los negocios transnacionales. Consejo General del Notariado. 
Fernández Rozas, J. C. (2007). Derecho de los Negocios internacionales. En Derecho de los negocios internacionales. Iustel.

Galgano, F. (2005). La globalización en el espejo del derecho. Santa Fe, Argentina: Rubinzal-Culcioni.

Garro, A. (1993). Armonización y unificación del derecho privado en América Latina: esfuerzos, tendencias y realidades. Centro di studi e ricerche di diritto comparato e straniero. Saggi, Conferenze e seminari, pp. 1 y 2. Recuperado de http://www.cisg. law.pace.edu/cisg/biblio/garro5.html

Gómez Vásquez, C. (2006). El principio de conservación del contrato en el derecho contractual internacional (análisis dogmático y cointextual). Ambiente jurídico. Universidad de Manizales. Recuperado de http://es.calameo. $\mathrm{com} / \mathrm{read} / 00032500554 \mathrm{ce} 3$ efebb72

González, A. (2004). La formación del contrato tras la ley de servicios de la sociedad de la información y de comercio electrónico. Granada: Comares.

Grün, E. (1995). Una visión sistémica y cibernética del derecho. Buenos Aires: Abeledo Perrot.

Illescas Ortiz, R., Perales Viscasillas, P. (2003). Derecho mercantil internacional. El derecho uniforme. Universidad Carlos III de Madrid. Ramón Areces.

Instituto Internacional para la Unificación del Derecho Privado (Unidroit). (2004). Principios Unidroit sobre los contratos comerciales internacionales. Recuperado de http:// www.unidroit.org/spanish/principles/contracts/principles2004/blackletter2004.pdf

Instituto Internacional para la Unificación del Derecho Privado (Unidroit). (2004). Principios Unidroit sobre los Contratos Co- merciales Internacionales. Recuperado de: http://www.unidroit.org/spanish/principles /contracts/principles2004/blackletter2004.pdf

Iriarte Ahon, E. (2003). Informe sobre Comercio electrónico en America Latina. Monitor de políticas TIC en América Latina $y$ el Caribe. Recuperado de http:/lac. derechos.apc.org/investigacion/comercio_electronico.doc

Iriarte Ahon, E. (2006). Comercio electrónico en América Latina: realidades y perspectivas. Monitor de políticas TIC en América Latina y el Caribe. Recuperado de http://lac.derechos.apc.org/investigacion/ comercio_electronico-1.pdf

Josserand, L. (1950). Teoría general de las obligaciones. Santiago Cunchillos y Manterola (trad.). Derecho civil. Tomo II. Vol. 1. Buenos Aires: Ediciones Jurídicas Europa América: Bosch y Cía.

Kelsen, H. (1995). Teoría pura del Derecho. Porrúa.

López Rodríguez, A. M. (2006). Las compilaciones orgánicas de principios generales del derecho de los contratos y su naturaleza jurídica. En Nueva lex mercatoria y contratos internacionales, tomo 2. Oviedo Albán, J.; Calvo Caravaca (directores). Colección Derecho Privado y Globalización. Ibáñez.

Madriñán de la Torre, R. E. (2000). Principios del derecho comercial, $6^{\mathrm{a}}$ edición. Santafé de Bogotá: Temis S. A.

Mantilla Espinosa, F. (2007). Sobre la formación del contrato. En Los contratos en el derecho privado. Bogotá: Universidad del Rosario: Legis.

Márquez, J. F. (2002). Elementos de la contratación electrónica. El acuse de recibo y la confirmación del mensaje. Anuario de de- 
recho civil. Universidad Católica de Córdoba. VII. Recuperado de http://www.acaderc. org.ar/doctrina/articulos/elementos-de-lacontratacion-electronica.-el-acuse

Martínez Gallego, E. (2000). La formación del contrato a través de la oferta y la aceptación. Madrid: Ed. Marcial Pons.

Mendoza Ramírez, A. (2008). Principios generales del derecho comercial. En Oviedo Albán, J. (coord.). Derecho comercial en el siglo XXI. Bogotá: Universidad de la Sabana: Temis.

Miranda Serrano, L. M., \& Pagador López, J. (2008). La formación y ejecución del contrato electrónico: aproximación a una realidad negocial emergente. Estudios sobre consumo. Universidad de Córdoba, 85, pp. 77-92. Recuperado de http:// helvia.uco.es/xmlui/bitstream/handle/10396/2239/Ec85_05.pdf?sequence=1

Moncayo, V. M. (2009). El leviatán deteriorado. Bogotá: Norma.

Misión Permanente de Estados Unidos de América ante la Organización de Estados Americanos (2002). Sexta Conferencia Especializada Internacional sobre Derecho Internacional Privado. Proyecto de reglas uniformes interamericanas en materia de documentos y firmas electrónicos.

Moreno Morejon, F. (s. f.). El perfeccionamiento de los contratos. Apuntes jurídicos. Recuperado de http://www. apuntesjuridicos.com.ec/download/noticias/723_DOC_OTRO.pdf

Newman Rodriguez, S. (2001). Aproximación a la formación del contrato electrónico en la legislación española. Revista Ventana Legal. Recuperado de http://www.ventanalegal.com/revista_ventanalegal/aproximacion.htm
Olivera, N. (2008). En busca de la lex retialis. Revista de derecho informático Alfa-Re$d i$. Recuperado de www.alfa-redi.org/rdiarticulo.shtml? $\mathrm{x}=10130$

Orduña Moreno, F. J. (2003). Derecho de la contratación y condiciones generales. En Homenaje a Luis Rojo Ajuria. Universidad de Cantabria. Recuperado de http:// books.google.com/books? $\mathrm{id}=\mathrm{nJXvmm} 4 \mathrm{r}$ $\mathrm{w} 6 \mathrm{YC} \&$ printsec $=$ frontcover\& $\mathrm{hl}=\mathrm{es} \# \mathrm{v}=\mathrm{o}$ nepage \&q\&f=false

Ospina Fernández, G., \& Ospina Fernández, E. (1994). Teoría general del contrato y de los demás actos o negocios jurídicos. Bogotá: Temis.

Oviedo Albán, J., Posada Núñez, L., \& Urbina Galiano, L. (1999). La formación del contrato en los principios de Unidroit para los contratos comerciales internacionales (Comparación con las normas colombianas). Vniversitas, 96. Pontifica Universidad Javeriana. Recuperado de http://www.cisg.law.pace.edu/cisg/biblio/ oviedoalban $1 . h t m l \# 8$

Oviedo Albán, J. (2001). Campo de aplicación y criterios de interpretación de la Convención de Viena para la compraventa internacional de mercaderías. Pace Law School Institute of International Commercial Law. Mayo de 2001. Recuperado de http://www.cisg.law.pace.edu/cisg/ biblio/alban.html\#7

Oviedo Albán, J. (2002). La unificación del derecho privado: Unidroit y los principios para los contratos comerciales internacionales. Pace Law School Institute of International Commercial Law.

Oviedo Albán, J. (2003). Transformaciones de la contratación mercantil: Hacia una nueva lex mercatoria. En Estudios de derecho económico, Tomo I. Instituciones de 
Derecho Comercial. Oviedo Albán, J.; García Muñoz, J. U. (coords.). Bogotá: Ibáñez.

Oviedo Albán, J. (2004). Aplicación directa de la Convención de Naciones Unidas sobre los contratos de compraventa internacional de mercaderías. Revista Vniversitas. Pontificia Universidad Javeriana, Facultad de Ciencias Jurídicas.

Oviedo Albán, J. (2007). Aplicación material de la Convención de Naciones Unidas sobre compraventa internacional de mercaderías. Universidad de la Sabana.

Oviedo Albán, J. (2008a). La formación del contrato. Bogotá: Universidad de la Sabana: Temis.

Oviedo Albán, J. (2008b). Regulación del contrato de compraventa internacional. Antecedentes, estructura y fuentes. Bogotá: Ibáñez.

Patroni Vizquerra, U. (2001). La contratación electrónica y el acuse de recibo. Teleley. Recuperado de http://www.teleley.com/ articulos/art-patroni.pdf

Peña Nossa, L. (2006). De los contratos mercantiles: nacionales e internacionales. Negocios del empresario. Bogotá: Universidad Católica de Colombia: ECOE. Recuperado de http://books.google.com/ books?id=_cM5bG7e8cC\&printsec $=\mathrm{fr}$ ontcover\& $\overline{\mathrm{h}} \mathrm{l}=\mathrm{es} \# \mathrm{v}=$ onepage $\& \mathrm{q} \& \mathrm{f}=$ false

Perales Viscasillas, M. P. (1998). La "batalla de los formularios" en la convención de Viena de 1980 sobre compraventa internacional de mercaderías: una comparación con la sección 2-207 UCC y los principios de Unidroit. Pace Law School Institute of International Commercial Law. Recuperado de http://www.cisg.law.pace.edu/cisg/ biblio/laley.html
Perales Viscasillas, M. P. (1999a). Formación. En Comentario a los principios Unidroit para los contratos del comercio internacional. Madrid: Aranzadi.

Perales Viscasillas, M. P. (1999b). El derecho uniforme del comercio internacional: los principios de Unidroit, p. 1. Recuperado de http://www.cisg.law.pace.edu/cisg/biblio/pcci.html\#nota*

Perales Viscasillas, M. P. (2001). El Contrato de Compraventa Internacional de Mercancías (Convención de Viena de 1980). Pace Law School Institute of International Commercial Law. Recuperado de http://www.cisg.law.pace.edu/cisg/biblio/ perales 1.html\#ci

Perales Viscasillas, M. P. (2009) Publicidad $\mathrm{y}$ formación del contrato. La formación electrónica del contrato en el Draft Common Frame of Reference. Revista de Contratación Electrónica, 100. Recuperado de http://vlex.com/vid/oacutenica-draft-frame-reference-57446028?ix resultado $=23.0 \&$ query $\% 5 \mathrm{Bbuscable}$ id $\% 5 \mathrm{D}=4 \&$ query $\% 5$ Bbuscable type $\%$ $5 \mathrm{D}=$ ColeccionTipo\&query $\% 5 \mathrm{Bphrase} \% 5$ $\mathrm{D}=$ formaci $\% \mathrm{C} 3 \% \mathrm{~B} 3 \mathrm{n}+\mathrm{del}+$ contrato

Pinochet Olave, R. (2005). La formación del consentimiento a través de las nuevas tecnologías de la información. Parte II: La aceptación electrónica. ¿Contratantes electrónicos, contratantes presentes o ausentes? Revista Ius et Praxis, 11, pp. 55-92. Recuperado de http://www.scielo.cl/scielo. php?pid=S071800122005000100004\&sc ript=sci_arttext

Planiol, M. \& Ripert, J. (1936). Las obligaciones (primera parte). En Tratado práctico de derecho civil francés. Tomo sexto. La Habana: Cultural S. A.

Remolina Angarita, N. (2006). Aspectos legales del comercio electrónico, la contratación 
y la empresa electrónica. Universidad de los Andes. Recuperado de http://gecti.uniandes.edu.co/docs/comercio $\% 20$ elect\%20Remolina.pdf

Rincón Cárdenas, E. (2004). Últimos retos para el derecho privado: las nuevas tecnologías de la información. Revista de Estudios socio-jurídicos. Recuperado de http://redalyc.uaemex.mx/redalyc/src/inicio/ArtPdfRed.jsp?iCve=73360215

Rubio Gimeno, G. (2003) Validez y perfección del contrato electrónico. En La regulación del comercio electrónico. Barral Viñals, I. (coord.). Madrid: Dykinson.

Serrano Acitores, A. (2010). El principio de conservación de los contratos frente a las figuras de la nulidad y la anulabilidad. Noticias Jurídicas. Recuperado de http:// noticias.juridicas.com/articulos/45-Derecho\%20Civil/201006-83473947392.html

Suescún Melo, J. (1996). Derecho privado. Estudios de derecho civil y comercial con- temporáneo. Bogotá: Universidad de los Andes.Cámara de comercio de Bogotá.

Superintendente Tributario General (2004). Resolución 00002-20004. Bolivia, 5 de julio.

Umaña Chaux, A. F. (2006). Contratación electrónica internacional. En Contratación Electrónica. Universidad del Rosario.

Vessuri, H. (2008). Tendencias de la Educación Superior en América Latina y el Caribe. En El futuro nos alcanza: mutaciones previsibles de la ciencia y la tecnología. Instituto Internacional de la Unesco para la Educación Superior en América Latina y el Caribe (Iesalc).

Zarur Miranda, X. (2008). Tendencias de la Educación Superior en América Latina y el Caribe. En Integración regional e internacionalización de la educación superior en América Latina y el Caribe. Instituto Internacional de la Unesco para la Educación Superior en América Latina y el Caribe (Iesalc). 